\title{
FIBRAS ALIMENTARES VERSUS OBESIDADE: O NUTRIENTE NÃO DIGERIDO NA PREVENÇÃO DO EXCESSO DE PESO
}

\author{
FOOD FIBERS VERSUS OBESITY: THE UNDIGESTED NUTRIENT IN PREVENTING \\ OVERWEIGHT
}

Melyssa de Souza e Silva Costa ${ }^{1}$

RESUMO: A obesidade vem ganhando destaque devido seu crescente aumento; e as funções das fibras já são observadas, como prevenção de implicações relacionadas à saúde. O objetivo desse estudo, foi investigar a atuação das fibras sobre o excesso de peso e obesidade. Utilizou-se a revisão integrativa da literatura, para o desenvolvimento desse estudo. Como resultado, foram incluídos no artigo 8 estudos que estavam em consonância com os critérios de inclusão/exclusão; os artigos discutem que as fibras podem atuar de maneira benéfica em complicações associadas ou não com a obesidade. Concluiu-se que a ingestão de fibras pode colaborar indiretamente na obesidade, devido sua atuação fisiológica e química, estimular o aumento da saciedade, redução da ingestão de energia, quelante de parte dos lipídios ingeridos e sobre a microbiota intestinal; diante do exposto, podem contribuir para déficit calórico e/ou absorção de energia. No entanto, há necessidade de mais estudos sobre o tema proposto.

Palavras-Chave: Fibras Alimentares. Obesidade. Nutrição.

ABSTRACT: Obesity has been gaining prominence due to its growing increase; and the functions of the fibers are already observed, as prevention of health-related implications. The aim of this study was to investigate the role of fibers on overweight and obesity. The integrative literature review was used to develop this study. As a result, studies that were in line with the inclusion / exclusion criteria were included in Article 8; the articles argue that fibers can act beneficially in complications associated or not with obesity. It was concluded that the ingestion of fibers can indirectly contribute to obesity, due to its physiological and chemical performance, to stimulate the increase of satiety, reduction of the energy intake, chelation of part of the ingested lipids and on the intestinal microbiota; in view of the above, they may contribute to calorie deficit and / or energy absorption. However, there is a need for further studies on the proposed theme.

Keywords: Dietary fibers. Obesity. Nutrition.

\footnotetext{
${ }^{1}$ Especialização em Nutrição Clínica. metabolismo. prática e terapia nutricional pela FAVENIFACULDADE VENDA NOVA DO IMIGRANTE, Brasil (2021)-Universidade Federal do Rio de Janeiro UFRJ.E-mail: melsouzanutri@gmail.com
} 


\section{INTRODUÇÃO}

A obesidade, pode contribuir para diversos malefícios à saúde; e nos últimos anos vem sendo crescente em todos os níveis sociais e despertando atenção por ser um problema de saúde pública (Dias e colaboradores, 2017); vislumbrando a promoção de melhora na qualidade de vida, em 2014 foi elaborado o guia alimentar para a população brasileira, cujo objetivo é incentivar práticas de alimentação saudável, descrevendo que a base da alimentação deve ser alimentos naturais e com o mínimo de processamento, expondo diversas razoes biopsicossociais e ambientais para o consumo desses alimentos como base (BRASIL, 2014).

A função das fibras alimentares nos últimos anos vem sendo levantada por diferentes autores; pois é notório que a ingestão de fibras na alimentação, estabelecem uma atuação sobre diversas doenças crônicas e ainda sobre o sistema imunológico. As fibras alimentares são resistentes as enzimas no trato gastrointestinal, são classificadas em solúveis, que absorvem água, formando géis de consistência viscosa, e são fermentáveis; e insolúveis, que não absorvem água, não formando, portanto, géis e não são fermentáveis; entretanto, as fibras solúveis podem ter relação com a diminuição da glicemia após a ingestão de carboidratos, o que pode ser devido ao fato da formação do gel retardar o esvaziamento gástrico (Bernaud e Rodrigues, 2013).

As fibras são discutidas por especialistas que sugerem atuação das mesmas sobre a obesidade, dentre os fatores relacionados, ressalta-se o fato das fibras não serem absorvidas, com isso não fornecendo calorias ao organismo; é notório também a necessidade de um maior tempo de mastigação, que pode gerar mais saciedade, formar géis que diminuem a velocidade do esvaziamento gástrico, aumentando assim a sensação de saciedade, além da possibilidade das fibras interagir como quelante de uma parte das gorduras, diminuindo a absorção e consequentemente a quantidade de calorias (Hurtado e Calliari, 2010) nesse sentido, essa revisão bibliográfica pode contribuir com achados na literatura que estabeleçam uma relação entre as fibras e a atuação das mesmas na prevenção obesidade, trazendo conhecimento sobre um problema de saúde pública atual e vislumbrando benefícios das fibras na alimentação nos indivíduos obesos. 
A revisão integrativa objetiva compilar resultado de pesquisas sobre alguma temática, de forma metódica e organizada; essa revisão da literatura é conhecida como integrativa por estabelecer uma forma mais ampla sobre um tema, o que permite ao autor a produção do conteúdo de revisão com diversos objetivos final (Ercole, Melo e Alcoforado, 2014); diante do exposto, foi utilizada a revisão integrativa da literatura para a elaboração deste estudo.

O estudo teve como objetivo geral investigar através da revisão integrativa da literatura se há uma correlação entre a atuação das fibras e a obesidade; e como objetivo específico, investigar as funções das fibras, conhecer as intercorrências da obesidade, o levantamento da literatura sobre os assuntos abordados, estabelecer correlação entre os assuntos e analisar os dados obtidos na literatura.

\section{MATERIAIS E MÉTODOS}

Esta pesquisa é uma revisão integrativa da literatura, que consiste em uma metodologia mais ampla, com fases definidas e que vem sendo aplicada no campo da saúde, para garantir um suporte com embasamentos científicos (Souza, Silva e Carvalho, 2010). A revisão integrativa percorre seis etapas para sua elaboração, a primeira diz respeito ao levantamento do assunto estudado, a segunda fase é a pesquisa na literatura científica, a terceira etapa é composta da coleta de dados, a quarta etapa é de avaliação da informação incluída no estudo, a quinta é a análise dos resultados e a sexta é um compilado da informação dos artigos analisados (Pompeo, Rossi e Galvão, 2009). A fase inicial dessa revisão bibliográfica compõe a elaboração da pergunta norteadora desse estudo: há correlação das fibras alimentares no controle da obesidade? A segunda e terceira fase da pesquisa, ocorrida em março de 202I, contemplou a investigação na literatura sobre a questão levantada; para isso ocorreram buscas nas bases de dados Literatura LatinoAmericana e do Caribe em Ciências da Saúde (LILACS) e Medical Literature Analysis and Retrieval System Online (MEDLINE) e para isso, utilizou-se como palavras-chave "fibras alimentares" e "obesidade", incluindo o operador booleano "and". Foram utilizados como critérios de inclusão, artigos em português, inglês e espanhol que abordassem a atuação das fibras alimentares na obesidade, do ano de 2015 a 2021; e excluídos artigos de acesso restrito e que não abordassem o tema. 
A inclusão dos artigos configurou a quarta etapa da pesquisa, expressa nos resultados; ocorreu a leitura analítica dos artigos incluídos e a discussão, conforme a etapa cinco e seis respectivamente.

Os processos da etapa de levantamento de dados estão demonstrados no fluxograma a seguir (Figura I).

FIGURA r. Fluxograma da coleta nas bases de dados.

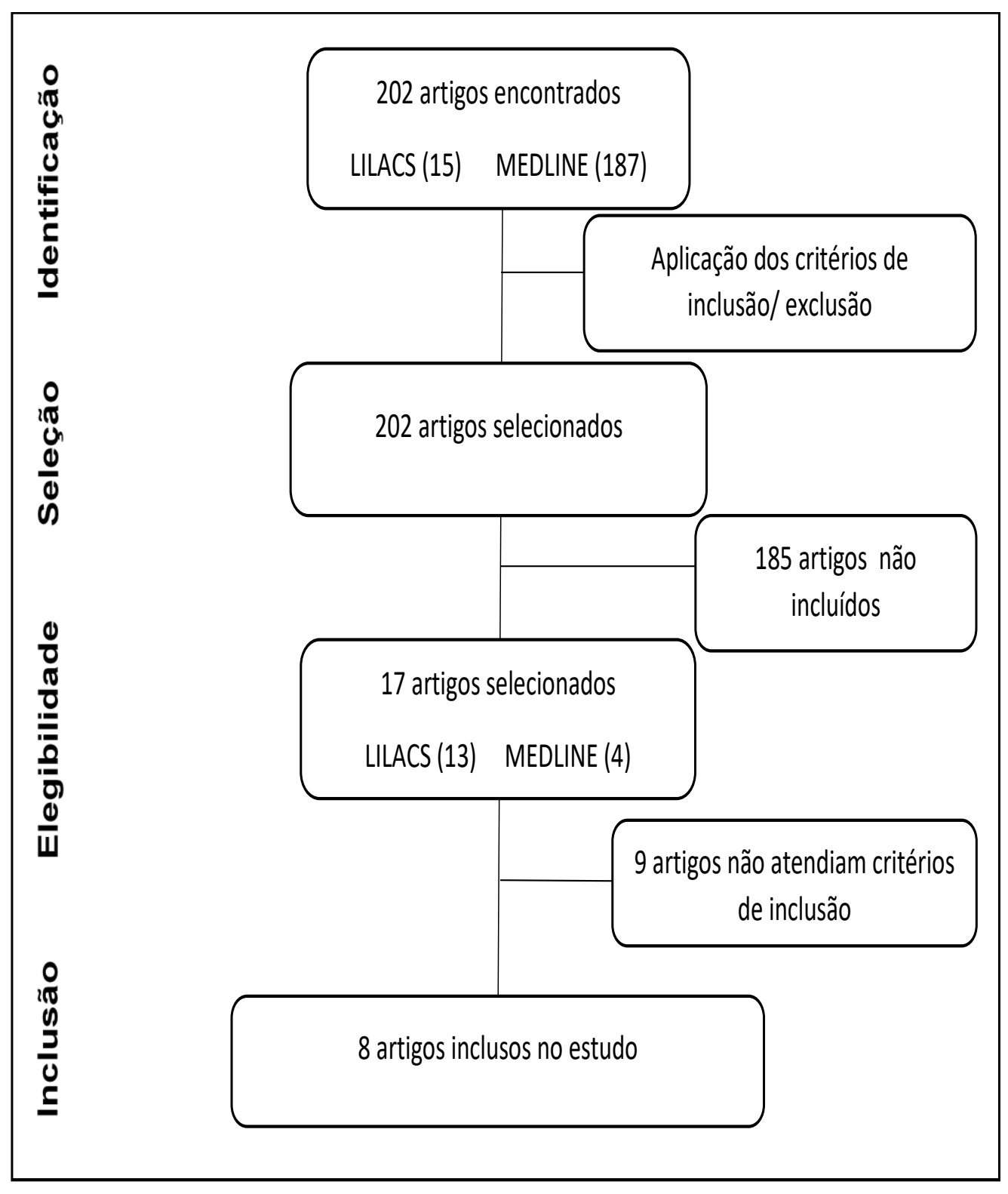

Fonte: Baseado em Galvão, Pansani e Harrad (2015)

\section{RESULTADOS}


Os artigos incluídos, foram selecionados, organizados e as principais características demonstradas (Tabela I)

FIGURA 2. Tabela das características dos artigos inclusos.

\section{Título}

Autores

Ano de Publicação

Tipo de Artigo

Combined Soluble Fiber-Mediated $\mathrm{Xu}$ et al.

Intestinal Microbiota Improve

Insulin Sensitivity of Obese Mice distinct non-starch polysaccharides

on fermentation, appetite

regulation and fat accumulation in

rat

Impact of dietary fiber Mayengba

supplementation on modulating met al.

microbiota-host-metabolicaxes in

obesity

Dietary Fibre as a Unifying Bozzeto et

Remedy for the Whole Spectrum

of Obesity-Associated

Cardiovascular Risk

Effects of isolated soluble fiber Tompson supplementation on body weight, et al.

glycemia, and insulinemia in adults

$$
\text { al. }
$$

2020

2019

2019

2018

Artigo Original

Estudo experimental

Artigo Original

Estudo Experimental
Artigo Original

Artigo de Pesquisa 
with overweight and obesity:a systematic review and metaanalysis of randomized controlled trials

\section{Título}

Ano

de Tipo de Artigo

Publicação

Intake and Dietary Food Sources of González-

Fibre in Spain:

Rodríguez

Differences with Regard to the et al.

Prevalence of Excess

Body Weight and Abdominal

Obesity in Adults of the ANIBES

Study

Bamboo shoot fiber prevents Li et al.

obesity in mice by modulating the

gut microbiota
2017

Artigo Original

Estudo de Pesquisa

Effects of Dietary Fibre (Pectin) Adam et

2016

\section{Artigo Original}

and/or

al.

Estudo experimental

Increased Protein (Casein or Pea)

on Satiety,

Body Weight, Adiposity and

Caecal

Fermentation in High Fat Diet-

Induced Obese Rats

Fonte: IBGE(1993). 
Foram encontrados inicialmente 202 artigos, destes 187 no MEDLINE e 15 LILACS, após critérios de inclusão e exclusão foram selecionados 13 artigos MEDLINE e 4 LILACS, os estudos foram lidos e inclusos 8 artigos, estes estavam de acordo com o tema proposto.

Dos artigos inclusos no estudo, 6 artigos são originais e 2 artigos contemplam revisão da literatura. Utilizaram uma metodologia experimental, em quatro estudos inclusos, que consiste em uma pesquisa in vivo, dois artigos apresentaram um estudo de pesquisa com humanos e dois são revisão da literatura.

\section{DISCUSSÃO}

Os mecanismos das fibras podem estar ligados à sua solubilidade, que consiste na capacidade de formar géis, diminuindo o esvaziamento gástrico e consequentemente, diminuindo a ingestão de energia através da alimentação; e pode também estar ligadas a capacidade de fermentação, produzindo ácido graxo de cadeia curta que equilibra a secreção de hormônios ligados à saciedade e controle do apetite, peptídeo- I (GLP-I) e grelina, essa fermentação pode atuar na oxidação lipídica e metabolismo da glicose (González-Rodriguez e colaboradores, 2017). O efeito da

fermentação das fibras produz ácido graxo de cadeia curta, contribuem para metabolismo de bactérias no intestino e fornecem uma parcela de aproximadamente I0\% de energia para nosso organismo; a suplementação da fibra de ervilha poderia apresentaram discreta modulação sobre a diversidade de bactérias intestinais e um possível efeito benéfico para evitar prejuízos sobre perfil metabólico (Mayengbam e colaboradores, 2019).

Os achados da literatura, sugerem que as fibras solúveis podem ser utilizadas na estratégia na dislipidemia, nesse sentido, a ingestão de fibras também pode ser benéficas nas doenças cardiovasculares e aliada a uma mudança no padrão alimentar saudável pode contribuir para evitar as doenças hepáticas não alcóolicas; entretanto, apesar de ainda não ser palpável o tipo de fibra na interferência sobre o metabolismo da glicose, no diabetes e na resistência à insulina, a ingestão de fibras precisa ser estimulada em indivíduos obesos (Bozzetto e colaboradores, 2018); a literatura, cita também, os efeitos benéficos da suplementação das fibras sobre perfil metabólico e antropométrico de indivíduos com excesso de peso e obesos (Thompson e colaboradores, 2017). 
A resistência à insulina pode ser um cofator de doenças metabólicas, e está ligada com o aumento da inflamação, seja a inflamação sistêmica ou crônica; e a suplementação de fibras nos camundongos, mesmo não sendo observados nenhum efeito visível da suplementação diretamente, demonstrou melhora da resistência à insulina, diversificação da microbiota intestinal e redução de marcadores da inflamação, o que ressalta a contribuição para o equilíbrio da obesidade (Xu e colaboradores, 2020).

A fibra do broto de bambu, foi evidenciada em um estudo, que concluiu dentre todas as fibras abordadas nas dietas dos camundongos, que as fibras do bambu obtiveram melhores resultados para evitar um aumento do peso; nesse sentido, houve o aprofundamento do estudo, que identificou diminuição do tamanho dos adipócitos dos camundongos que receberam alimentação enriquecida com a fibra do bambu; no estudo também houve ênfase da influência das fibras na modulação intestinal, visto que, a uma microbiota intestinal diversificada podem contribuir para evitar o aumento de peso (Li e colaboradores, 2016).

As fibras solúveis ganham destaque no que se refere a sua funcionalidade, em uma pesquisa in vivo, analisou a modulação da pectina do farelo de soja e a betaglucana presente na aveia, e concluíram, de forma inédita, que o butirato e o acetato, sendo os níveis e as quantidades de butirato inversamente proporcional ao peso e os níveis de acetato proporcional ao peso, o estudo sugere que a suplementação pode ser um bom manejo da obesidade, visto que aumentou as concentrações de butirato nos camundongos (Tian e colaboradores, 2019). Nessa mesma direção, as dietas suplementadas com pectina em camundongos obtiveram mais eficácia do que as dietas com proteínas, porém, estudos que abordam a investigação de dietas ricas em fibras quando comparadas a dietas ricas em proteínas para a perda de peso necessitam de maiores investigação em humanos, entretanto, a pectina obteve maior desempenho em relação a adiposidade corporal, indicando que o ganho de peso nos ratos está diretamente ligado ao aumento da saciedade, que consequentemente diminui a ingestão de alimentos (Adam e colaboradores, 2016).

\section{CONCLUSÃO}

A relevância das fibras sobre a resistência à insulina, dislipidemias, doenças cardiovasculares e microbiota intestinal, são discutidas em diversos estudos da literatura, configurando assim a notoriedade das fibras na promoção de saúde. Em relação a 
prevenção da obesidade, concluiu-se que a ingestão de fibras podem colaborar indiretamente sobre o peso corporal; isso devido as funções fisiológicas e bioquímicas que as fibras desempenham, dentre elas, o estímulo ao aumento da saciedade, sua função como quelante de parte dos lipídios ingeridos, além de não fornecer calorias; contribuindo assim para redução da ingestão ou absorção de energia, sugerindo papel positivo no controle do peso, no entanto, há necessidade de mais estudos envolvendo a temática.

\section{REFERÊNCIAS}

ADAM, Clare L. et al. Effects of Dietary Fibre (Pectin) and/or Increased Protein (Casein or Pea) on Satiety, Body Weight. Plos One, Western University Of Health Sciences, Eua, v. II, n. 5, p. I-I6, 25 maio 20I6. Public Library of Science (PLoS). http://dx.doi.org/Io.1371/journal.pone.0155871.

BRASIL, Ministério da saúde. Guia alimentar para a População Brasileira. 2. ed. Brasil: Ms, 2014. $156 \mathrm{p}$.

BERNAUD, Fernanda Sarmento Rolla; RODRIGUES, Ticiana C.. Fibra alimentar: ingestão adequada e efeitos sobre a saúde do metabolismo. Arquivos Brasileiros de Endocrinologia \& Metabologia, Porto Alegre, Rs, v. 57, n. 6, p. 397-405, ago. 2013. FapUNIFESP (SciELO). http://dx.doi.org/10.1590/s0004-27302013000600oor.

BOZZETTO, Lutgarda et al. Dietary Fibre as a Unifying Remedy for the Whole Spectrum of Obesity-Associated Cardiovascular Risk. Nutrients, Nápolis, Itália, v. ıo, n. 7, p. o-943, 21 jul. 2018. MDPI AG. http://dx.doi.org/ro.3390/nuroo70943.

DIAS, Patricia Camacho et al. Obesidade e políticas públicas: concepções e estratégias adotadas pelo governo brasileiro. Cadernos de Saúde Pública, Niterói, Rj, v. 33, n. 7, p. I-I2, 2017. FapUNIFESP (SciELO). http://dx.doi.org/ro.159o/oro2-3ıгxoooo6or6.

ERCOLE, Flávia Falci; MELO, Laís Samara de; ALCOFORADO, Carla Lúcia Goulart Constant. Integrative review versus systematic review. Reme: Revista Mineira de Enfermagem, Belo Horizonte, Mg, v. I8, n. I, p. o9-II, 20I4. GNi Genesis Network. http://dx.doi.org/10.5935/1415-2762.201400oI.

GALVÃO, Taís Freire; PANSANI, Thais de Souza Andrade; HARRAD, David (org.). Principais itens para relatar Revisões sistemáticas e Meta-análises: a recomendação prisma. Epidemiologia e Serviços de Saúde, Brasília, v. 24, n. 2, p. 335-342, jun. 2015. FapUNIFESP (SciELO). http://dx.doi.org/10.5123/s1679-49742015000200o17. 
GONZÁLEZ-RODRÍGUEZ, Liliana et al. Intake and Dietary Food Sources of Fibre in Spain: differences with regard to the prevalence of excess body weight and abdominal obesity in adults of t. Nutrients, Espanha, v. 9, n. 4, p. 0-326, 25 mar. 2017. MDPI AG. http://dx.doi.org/10.3390/nu9040326.

HURTADO, Daiane Cristina; CALLIARI, Caroline Maria. FIBRAS ALIMENTARES NO CONTROLE DA OBESIDADE. Inesul, Londrina, v. o, n. o, p. o-I5, jan. 2010.

IBGE. Normas de Apresentação Tabular. 3. ed. Rio de Janeiro: Centro de Documentação e Disseminação da Informação, 1993.62 p.

LI, Xiufen et al. Bamboo shoot fiber prevents obesity in mice by modulating the gut microbiota. Scientific Reports, China, v. 6, n. I, p. I-II, 7 set. 20I6. Springer Science and Business Media LLC. http://dx.doi.org/10.1038/srep32953.

MAYENGBAM, Shyamchand et al. Impact of dietary fiber supplementation on modulating microbiota-host-metabolic axes in obesity. The Journal Of Nutritional Biochemistry, Canadá, v. 64, p. 228-236, fev. 2019. Elsevier BV. http://dx.doi.org/ı.1016/j.jnutbio.2018.11.003.

POMPEO, Daniele Alcalá; ROSSI, Lídia Aparecida; GALVÃO, Cristina Maria. Revisão integrativa: etapa inicial do processo de validação de diagnóstico de enfermagem. Acta Paulista de Enfermagem, São Paulo, v. 22, n. 4, p. 434-438, 2009. FapUNIFESP (SciELO). http://dx.doi.org/10.1590/so103-21002009000400014.

SOUZA, Marcela Tavares de; SILVA, Michelly Dias da; CARVALHO, Rachel de. Integrative review: what is it? how to do it? Einstein (São Paulo), São Paulo, v. 8, n. I, p. I02-106, mar. 2010. FapUNIFESP (SciELO). http://dx.doi.org/10.159o/si67945082010 rwil34.

THOMPSON, Sharon V; A HANNON, Bridget; AN, Ruopeng; HOLSCHER, Hannah D. Effects of isolated soluble fiber supplementation on body weight, glycemia, and insulinemia in adult: a systematic review and meta-analysis of randomized controlled trials. The American Journal Of Clinical Nutrition, Estados Unidos, v. Io6, n. 6, p. I5141528, I nov. 2017. Oxford University Press (OUP). http://dx.doi.org/ı0.3945/ajcn.117.163246. 
TIAN, Lingmin et al. Effect of oat and soybean rich in distinct non-starch polysaccharides on fermentation, appetite regulation and fat accumulation in rat. International Journal of Biological Macromolecules, [S.L.], v. 140, p. 515-521, nov. 2019. Elsevier BV. http://dx.doi.org/ı.1o16/j.ijbiomac.2019.o8.032.

$\mathrm{XU}$, Chuanhui et al. Combined Soluble Fiber-Mediated Intestinal Microbiota Improve Insulin Sensitivity of Obese Mice. Nutrients, China, v. I2, n. 2, p. 0-351, 29 jan. 2020. MDPI AG. http://dx.doi.org/ro.3390/nui2020351. 